\title{
Compact and broadband polarization beam splitter based on a silicon nitride augmented low-index guiding structure
}

\author{
X. Sun, ${ }^{1, *}$ M. Z. Alam, ${ }^{2}$ J. S. Aitchison, ${ }^{1}$ and M. Mojahed ${ }^{1}$ \\ ${ }^{1}$ Department of Electrical \& Computer Engineering, University of Toronto, 10 King's College Road, Toronto, Ontario M5S3G4, Canada \\ ${ }^{2}$ Department of Applied Physics and Materials Science, California Institute of Technology, Pasadena, California 91106, USA \\ *Corresponding author: xiaoxiao.sun@utoronto.ca
}

Received 21 October 2015; accepted 7 November 2015; posted 24 November 2015 (Doc. ID 251722); published 22 December 2015

We propose a compact polarization beam splitter (PBS) based on a silicon nitride enhanced silicon-on-insulator platform using an augmented low-index guiding (ALIG) waveguide structure. In the ALIG structure, the TM mode is mostly confined in the low-index silicon nitride, while the TE mode is confined in the high-index silicon. Since the two modes are confined in two separate layers, their properties can be controlled independently. The PBS is formed using an asymmetric multimode interference (MMI) section. The TM mode is directed to an output port by the ALIG waveguide, while the TE mode is coupled to the other output port via the multimode interferometer. Such a PBS has a very small footprint, low insertion loss, high polarization extinction ratio, and broadband response. () 2015 Optical Society of America

OCIS codes: (130.2790) Guided waves; (130.3120) Integrated optics devices; (130.5440) Polarization-selective devices; (230.7370) Waveguides.

http://dx.doi.org/10.1364/OL.41.000163

In recent years, photonic integrated circuits have attracted much interest [1]. One of the most promising platforms is based on high refractive index contrast waveguides; however, this highindex contrast also introduces a large polarization sensitivity. This may cause significant problems when light is coupled to a chip from an optical fibers since the polarization state of the light in such fibers can vary randomly. Consequently, there is a need for compact and broadband polarization control elements to manipulate and track the polarization state of the optical signal on the chip. One approach is to use a polarization diversity scheme consisting of polarization beam splitters and polarization rotators [2]. The polarization beam splitter (PBS) can separate the transverse electric (TE) and transverse magnetic (TM) polarizations into different waveguides. Many different types of onchip PBSs have been proposed based on different mechanisms. A Mach-Zehnder interferometer PBS requires very long arms to achieve a relative $\pi$ phase shift between the TE and TM modes [3]. Mode evolution-based PBS also requires a very long device length to accomplish mode coupling and mode evolution [4]. While directional coupler-based PBSs have the advantage of small footprints and high extinction ratios (ER), they are sensitive to wavelength changes and have low fabrication tolerances [5-9]. Some PBSs take advantage of plasmonics via using silver pillars or nano ribbons, but they suffer from additional insertion losses due to the metal absorption and require high aspect ratio fabrication techniques [10,11]. Multimode interference (MMI) based PBS is another popular option which offers the benefit of low insertion loss and broadband response [12-15]. The existence of different beat lengths for the TE and TM modes is usually used in the design of MMI-based PBS; however, because the polarization dependence of the beat length is low, to effectively separate the TE and TM modes, an MMI section that is tens of microns long is needed [12-14]. Last, a very short PBS based on the hybrid plasmonic waveguides was proposed in [15], but the structure is difficult to fabricate, and the quality of the deposited metal can degrade the performance of the device.

In this Letter, we propose an ultra-compact PBS based on a new structure, the augmented low-index guiding (ALIG) waveguide $[16,17]$. Such a waveguide is based on a silicon nitride enhanced silicon-on-insulator platform. It consists of a low-index substrate (silica), a low-index cladding layer (air or silica), and a two-layer core. The core is made up of two dielectric materials with a high-index contrast (silicon and silicon nitride), as shown in Fig. 1. In this structure, the TE mode is guided in the highindex silicon waveguide whereas, by controlling the waveguide dimensions, the TM mode can be confined in the lower index silicon nitride layer.

For the structure in Fig. 1, with a silicon thickness equal to $220 \mathrm{~nm}$, light is guided by the silicon layer using the total internal reflection as in the case of a conventional silicon

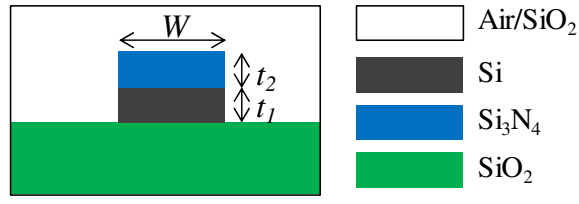

Fig. 1. Schematic cross section of the augmented low-index guiding (ALIG) waveguide. 
waveguide. However, if the silicon layer is thin enough (for example, $t_{1}=120 \mathrm{~nm}$ ), the TM mode leaks to the substrate with an air cladding, or is very loosely guided in the case of silica cladding and, thus, the two layers (silicon and silicon nitride) work together as the waveguide core. Typical field intensity profiles for the TM and TE modes at $1.55 \mu \mathrm{m}$ in the ALIG waveguide with silica cladding are simulated using Lumerical MODE Solutions and are shown in Figs. 2(a) and 2(b). The waveguide dimensions are $t_{1}=120 \mathrm{~nm}, t_{2}=350 \mathrm{~nm}$, and $W=600 \mathrm{~nm}$. For the TM mode, the normal component of the electric field shows a discontinuity at the $\mathrm{Si}_{3} \mathrm{~N}_{4} / \mathrm{Si}$ interface; thus, the field amplitude is much higher in the lower index region and electric field amplitude in the silicon nitride layer is dramatically increased. Since the dominant electric field for the TE mode is tangential to the $\mathrm{Si}_{3} \mathrm{~N}_{4} / \mathrm{Si}$ interface, there is no discontinuity in the electric field, and the TE mode is well guided by the thin silicon layer.

From Fig. 2(b), it is clear that most of the power for the TE mode resides in the silicon layer as a conventional waveguide. However, for the TM mode, with proper choice of waveguide dimensions, most of the power can reside in the silicon nitride layer, as shown in Fig. 2(a). Figure 3 shows a more detailed analysis of the power distribution in different layers for the TM mode of the ALIG waveguide for different silicon nitride thicknesses. The silicon thickness $\left(t_{1}\right)$ is optimized to $120 \mathrm{~nm}$, and the waveguide width $(W)$ is optimized to $600 \mathrm{~nm}$. For these dimensions, the waveguide is single mode. As the figure indicates, for properly chosen silicon nitride thickness (for example, $t_{2}=350 \mathrm{~nm}$ ), the power inside the nitride layer can exceed $60 \%$, while the power inside the silicon layer is approximately $9 \%$.

As stated earlier, in the ALIG waveguide the TE and TM modes reside in two different layers. Consequently, this platform can be used for on-chip polarization control. Moreover, unlike the hybrid plasmonic platform, the ALIG waveguides are purely dielectric and, hence, have lower propagation losses. In the following, we propose a PBS based on the ALIG waveguide using a MMI structure.
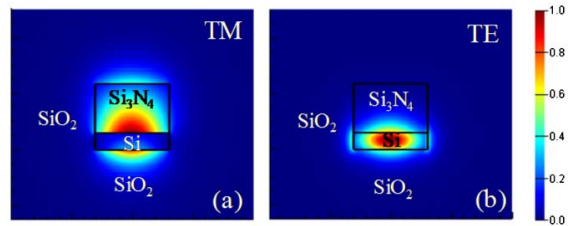

Fig. 2. Intensity profile in the ALIG waveguide with silica cladding for (a) TM mode and (b) TE mode. The operational wavelength is $1.55 \mu \mathrm{m}$

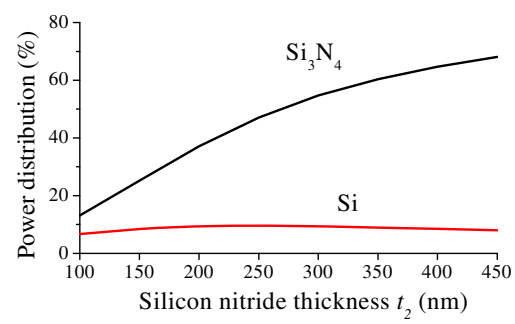

Fig. 3. TM power distribution inside the ALIG waveguide with $t_{1}=120 \mathrm{~nm} ; W=600 \mathrm{~nm}$ for different silicon nitride thicknesses $\left(t_{2}\right)$.

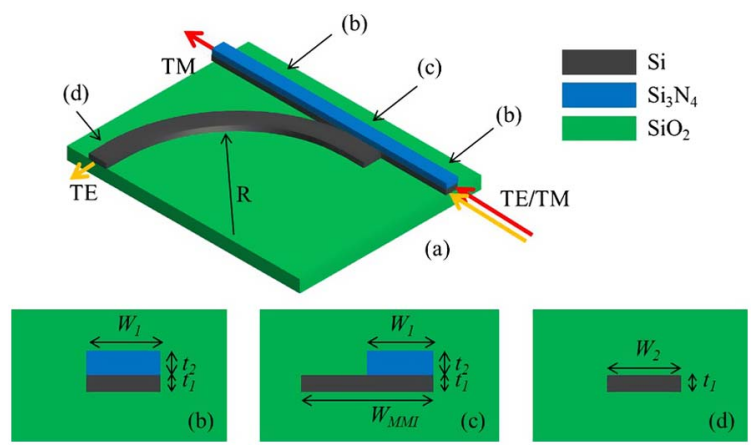

Fig. 4. (a) Three-dimensional schematic of the PBS. (b) Cross section of the input waveguide (supporting both TE and TM modes) and the output waveguide for the TM mode. (c) Cross section of the MMI segment. (d) Cross section of the output waveguide for the TE mode.

Figure 4(a) shows the three-dimensional schematic of the proposed PBS. The device consists of an input waveguide [Fig. 4(b)], an asymmetric MMI section [Fig. 4(c)], and output waveguides [(Figs. 4(b) and 4(d)] for different polarizations. The proposed PBS works with either air or silica claddings. Here, considering the packaging requirements of integrated photonics circuits, a silica cladding is chosen. The input and output waveguides for the TM branch have the same structure, which is shown in Fig. 4(b). For these input and output waveguides, the thicknesses of silicon and silicon nitride layers are $t_{1}$ and $t_{2}$, respectively, and the waveguide width is $W_{1}$. The cross section of the asymmetric MMI section is shown in Fig. 4(c). In the MMI section, the width of the silicon layer changes from $W_{1}$ to $W_{\mathrm{MMI}}$, while all other dimensions remain the same. Finally, as shown in Fig. 4(d), a silicon waveguide with the width $W_{2}$ and bend radius $R$ is used to guide the TE mode out of the MMI section.

The thickness of the silicon $t_{1}=120 \mathrm{~nm}$ and the thickness of the silicon nitride $t_{2}=350 \mathrm{~nm}$ are optimized for the ALIG structure. To keep the overall dimensions of the device small, the width of the input waveguide $\left(W_{1}\right)$ is set to $400 \mathrm{~nm}$. At this stage of the design, we need to optimize the width of the MMI section $\left(W_{\mathrm{MMI}}\right)$. Since the mode distributions for the TE and TM modes in the ALIG waveguide are different, the changing of the width of the silicon layer has little impact on the TM mode, while it affects the TE mode dramatically. Figure 5 shows the calculated effective indices of the eigenmodes in the MMI section. The effective indices for the TM modes are almost constant for all the widths, while the effective indices of the TE modes increase quickly with increasing $W_{\mathrm{MMI}}$. For

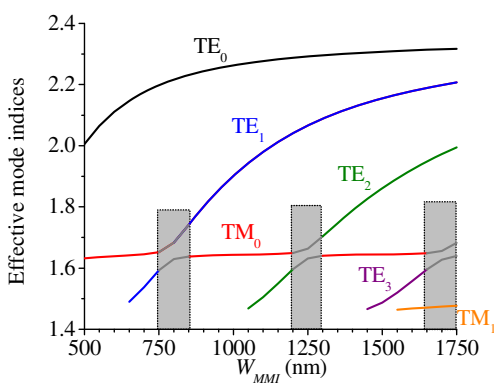

Fig. 5. Effective indices for the modes in asymmetric MMI waveguide for different MMI widths ( $W_{\text {MMI }}$ ). 
$W_{\text {MMI }}$ up to $1.2 \mu \mathrm{m}$, only the fundamental TM mode exits and no higher-order TM modes can be excited. At the same time, when the silicon is wide enough to support at least two TE modes, the mirror image in the MMI section for the TE modes can be formed. It should be noted that, for some values of the $W_{\text {MMI }}$ (grey regions in Fig. 5), the effective indices of two modes (fundamental TM and higher-order TE) approach each other; thus, the polarization state of these eigenmodes cannot be distinguished. This is due to mode hybridization, and light propagating in a waveguide with such dimensions will encounter mode conversion similar to the mode conversions in silicon ridge waveguides as discussed in [18]. These dimensions should be avoided in the design.

To maintain the device compactness, we choose $W_{\mathrm{MMI}}=$ $1 \mu \mathrm{m}$. The device with this width supports two TE modes $\left(\mathrm{TE}_{0}\right.$ and $\left.\mathrm{TE}_{1}\right)$ and only one TM mode $\left(\mathrm{TM}_{0}\right)$; it allows us to separate and direct the two polarizations to two different output waveguides. The calculated electric field profiles of the MMI section with $W_{\text {MMI }}=1 \mu \mathrm{m}$ are shown in Fig. 6 . The fundamental and the first-order TE modes are shown in Figs. 6(a) and 6(b), respectively. The fundamental TM mode is shown in Fig. 6(c), where no higher-order TM mode is supported.

The length of the MMI section $\left(L_{\mathrm{MMI}}\right)$ is chosen such that a mirror image for the TE mode is formed at the opposite port. The final dimensions are $t_{1}=120 \mathrm{~nm}, t_{2}=350 \mathrm{~nm}, W_{1}=$ $400 \mathrm{~nm}, W_{2}=600 \mathrm{~nm}, W_{\mathrm{MMI}}=1 \mu \mathrm{m}, L_{\mathrm{MMI}}=1 \mu \mathrm{m}$, and $R=5 \mu \mathrm{m}$. Guided TM and TE powers through the device for the TM and TE modes are simulated using Lumerical FDTD, as shown in Figs. 7(a) and 7(b), respectively. The TM mode is mainly guided by the silicon nitride layer to the straight output port, while the TE mode is transferred to the other output port due to the multimode interference.

Transmissions for the TM and TE modes at different output ports are calculated using Lumerical FDTD and are shown in Fig. 8(a). The insertion loss of the TM mode is only $0.3 \mathrm{~dB}$, and the insertion loss for the TE mode is less than $0.8 \mathrm{~dB}$. The corresponding Extinction Ratios (ERs), defined as the ratio of power for one mode to the other mode in the corresponding
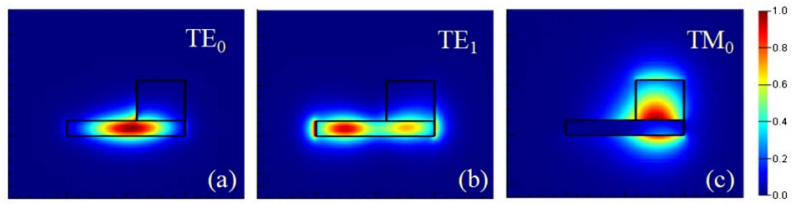

Fig. 6. Field distributions inside the asymmetric MMI waveguide for (a) $\mathrm{TE}_{0}$, (b) $\mathrm{TE}_{1}$, and (c) $\mathrm{TM}_{0}$. The operating wavelength is $1.55 \mu \mathrm{m}$; dimensions are $t_{1}=120 \mathrm{~nm}, t_{2}=350 \mathrm{~nm}, W_{1}=400 \mathrm{~nm}$, $W_{2}=600 \mathrm{~nm}$, and $W_{\mathrm{MMI}}=1 \mu \mathrm{m}$.
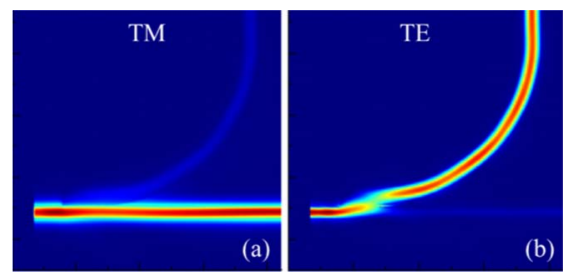

Fig. 7. Light propagates along the proposed PBS for the (a) TM mode and (b) TE mode at $1.55 \mu \mathrm{m}$. Device dimensions are given in the text.
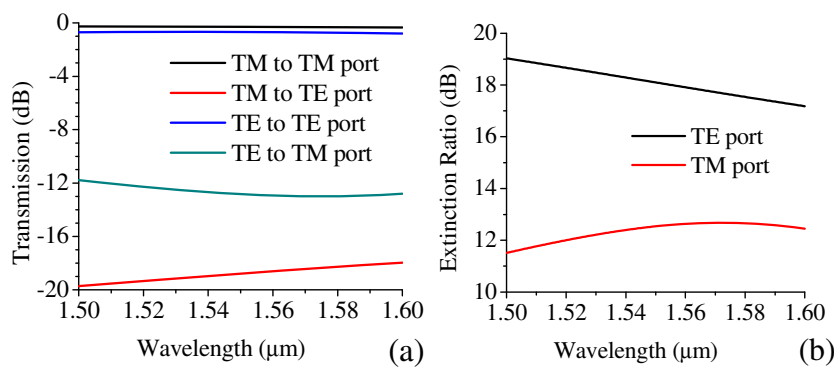

Fig. 8. (a) Transmission and (b) extinction ratio (ER) for the TE and TM output ports for the PBS with $t_{1}=120 \mathrm{~nm}, t_{2}=350 \mathrm{~nm}$, $W_{1}=400 \mathrm{~nm}, W_{2}=600 \mathrm{~nm}, W_{\mathrm{MMI}}=1 \mu \mathrm{m}$, and $L_{\mathrm{MMI}}=$ $1 \mu \mathrm{m}, R=5 \mu \mathrm{m}$.

output port, are shown in Fig. 8(b). Over a bandwidth of $100 \mathrm{~nm}$, the ER for the TE output port is $17.2-19 \mathrm{~dB}$, and the ER for the TM output port is $11.5-12.6 \mathrm{~dB}$.

The results in Fig. 8 show that the proposed PBS is compact and broadband, with low insertion loss and an ER that is comparable to a short MMI-PBS based on the hybrid plasmonic waveguide [15]. Since the proposed PBS is very compact, we can further increase its performance with a larger MMI section. A wider MMI section offers a better separation for the TE and TM modes while simultaneously increasing the ERs. A schematic of the modified MMI section is provided in Fig. 9. We choose $W_{\mathrm{MMI}}=1.6 \mu \mathrm{m}$, which supports more TE modes. Accordingly, the input waveguide can be wider and is modified to be $W_{1}=450 \mathrm{~nm}$. To better transition to the TE mode, a $2 \mu \mathrm{m}$ long taper $\left(L_{a}=2 \mu \mathrm{m}\right)$ is proposed to change the silicon width from 450 to $900 \mathrm{~nm}$ at the input side. This taper helps the distribution of the TE mode to be more symmetric and to form a clearer mirror image in the asymmetric MMI section. The mirror image for the TE input forms at $L_{\mathrm{MMI}}=4.8 \mu \mathrm{m}$. At the output side, a $1 \mu \mathrm{m}$ long taper $\left(L_{b}=1 \mu \mathrm{m}\right)$, transitioning from 1 to $600 \mathrm{~nm}$ wide, is also used to collect more power for the TE mode which is then connected to a bend at the output side. The output taper is separated from the ALIG waveguide using a $100 \mathrm{~nm}$ gap. The final device dimensions are $t_{1}=120 \mathrm{~nm}, t_{2}=350 \mathrm{~nm}, W_{1}=450 \mathrm{~nm}$, $W_{2}=600 \mathrm{~nm}, W_{\mathrm{MMI}}=1.6 \mu \mathrm{m}, L_{\mathrm{MMI}}=4.8 \mu \mathrm{m}, W_{a}=$ $900 \mathrm{~nm}, L_{a}=2 \mu \mathrm{m}, W_{b}=1 \mu \mathrm{m}, L_{b}=1 \mu \mathrm{m}$, and $R=$ $5 \mu \mathrm{m}$.

For the modified structure with $W_{\mathrm{MMI}}=1.6 \mu \mathrm{m}$, now four TE and two TM modes are supported in the MMI section. The calculated electric field profiles of the modified MMI section are shown in Fig. 10. Although both $\mathrm{TM}_{0}$ and $\mathrm{TM}_{1}$ modes exist, due to the very small mode overlap, the mode

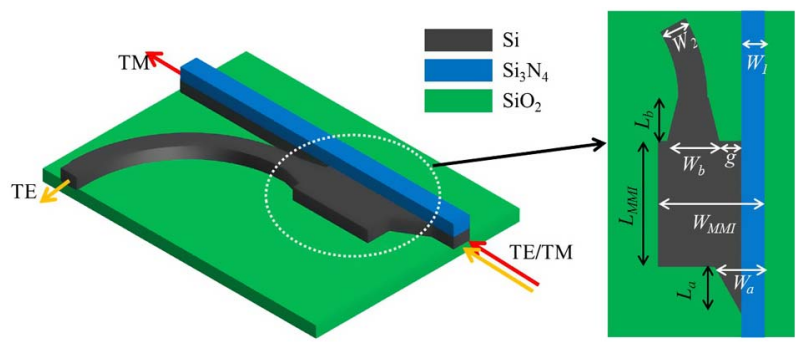

Fig. 9. Three-dimensional schematic of the modified PBS and top view of the MMI section. 

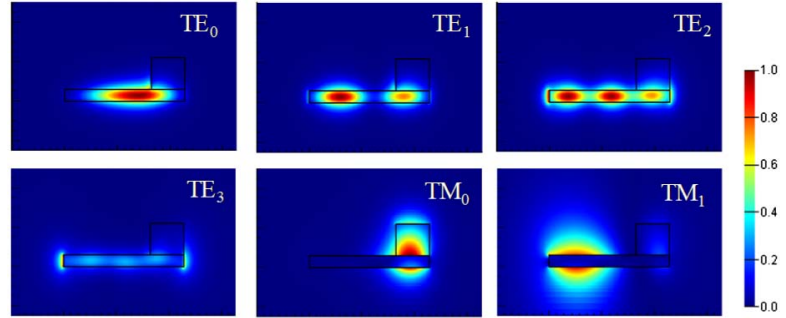

Fig. 10. Field distributions inside the asymmetric MMI waveguide for the modified PBS. The operating wavelength is $1.55 \mu \mathrm{m}$; dimensions are $t_{1}=120 \mathrm{~nm}, t_{2}=350 \mathrm{~nm}, W_{1}=450 \mathrm{~nm}, W_{2}=600 \mathrm{~nm}$, and $W_{\mathrm{MMI}}=1.6 \mu \mathrm{m}$.
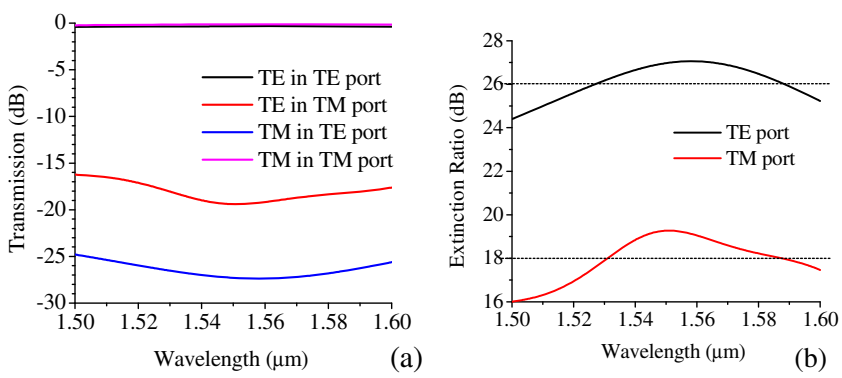

Fig. 11. (a) Transmissions and (b) extinction ratios for the TE and TM output ports of the modified PBS with $t_{1}=120 \mathrm{~nm}, t_{2}=$ $350 \mathrm{~nm}, \quad W_{1}=450 \mathrm{~nm}, W_{2}=600 \mathrm{~nm}, \quad W_{\mathrm{MMI}}=1.6 \mu \mathrm{m}$, $L_{\mathrm{MMI}}=4.8 \mu \mathrm{m}, W_{a}=900 \mathrm{~nm}, L_{a}=2 \mu \mathrm{m}, W_{b}=1 \mu \mathrm{m}, L_{b}=$ $1 \mu \mathrm{m}$, and $R=5 \mu \mathrm{m}$.

excitation ratio from the input fundamental TM mode $\left(\mathrm{TM}_{0}\right)$ to the higher-order TM mode $\left(\mathrm{TM}_{1}\right)$ is very low. Moreover, the $\mathrm{TM}_{1}$ mode is very leaky and not well guided by the ALIG waveguide. These imply that the presence of the higher-order $\mathrm{TM}_{1}$ mode has little impact on the operation of the PBS, which can also be attributed to the fact that the $\mathrm{TM}_{0}$ mode is well confined and guided by the silicon nitride layer.

The transmissions for the TM and TE modes of the modified PBS are shown in Fig. 11(a), and the corresponding ERs are shown in Fig. 11(b). The ER for the PBS with the wider MMI section has been improved: for a bandwidth over $100 \mathrm{~nm}$, the ER for the TE port is more than $24 \mathrm{~dB}$, and the ER for the TM port is more than $16 \mathrm{~dB}$. Moreover, the corresponding ERs for the TE and TM ports are more than 26 and $18 \mathrm{~dB}$ for the C-band $(1.53-1.565 \mu \mathrm{m})$, respectively. The insertion losses for both modes are less than $0.4 \mathrm{~dB}$ over the $100 \mathrm{~nm}$ bandwidth.

We have also analyzed the PBS for fabrication tolerances. The most import parameters are the width and length of the MMI section. The ERs for slightly varied MMI section are calculated and shown in Fig. 12. Figure 12(a) depicts the ERs for $L_{\mathrm{MMI}}=4.8 \mu \mathrm{m} \pm 50 \mathrm{~nm}$, while Fig. 12(b) shows the ERs for $W_{\mathrm{MMI}}=1.6 \mu \mathrm{m} \pm 25 \mathrm{~nm}$. These tolerances are quite enough for fabrication purposes.

In summary, we have analyzed the properties of the ALIG waveguide, which is based on the silicon nitride enhanced silicon-on-insulator platform. The ALIG waveguide shows very
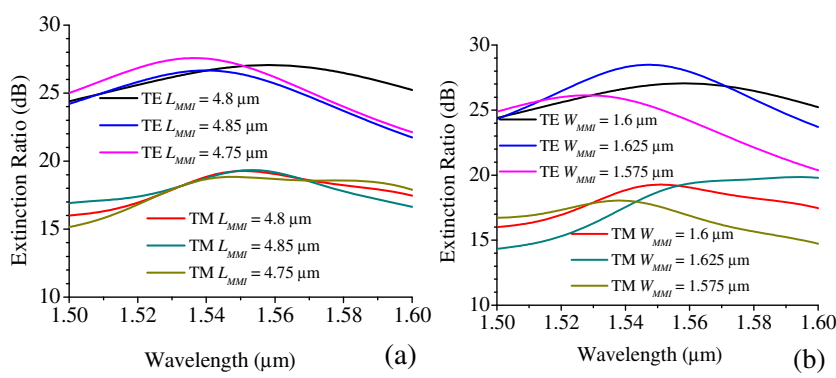

Fig. 12. Extinction ratio for the $\mathrm{TM}$ and $\mathrm{TE}$ ports with $t_{1}=120 \mathrm{~nm}, \quad t_{2}=350 \mathrm{~nm}, W_{1}=450 \mathrm{~nm}, \quad W_{2}=600 \mathrm{~nm}$. (a) $W_{\mathrm{MMI}}=1.6 \mu \mathrm{m}$ and $L_{\mathrm{MMI}}=4.75 / 4.8 / 4.85 \mu \mathrm{m}$. (b) $L_{\mathrm{MMI}}=$ $4.8 \mu \mathrm{m}$ and $W_{\mathrm{MMI}}=1.575 / 1.6 / 1.625 \mu \mathrm{m}$.

different mode confinements for the two orthogonal polarizations. We have proposed a PBS by utilizing an asymmetric MMI coupler based on the ALIG structure. The PBS could be extremely compact with an MMI section of only $1 \times 1 \mu \mathrm{m}$, and better performances can be achieved for the device with a $1.6 \times$ $4.8 \mu \mathrm{m}$ MMI section. Over a bandwidth of $100 \mathrm{~nm}$, the PBS with the $1.6 \times 4.8 \mu \mathrm{m}$ MMI section has a low insertion loss of less than $0.4 \mathrm{~dB}$ for both TM and TE modes. The polarization ERs over the whole C-band are $2618 \mathrm{~dB}$ for the TE and TM modes, respectively. The proposed PBS also shows good tolerance to fabrication uncertainties.

Funding. Natural Sciences and Engineering Research Council of Canada (NSERC).

\section{REFERENCES}

1. D. Dai, J. Bauters, and J. E. Bowers, Light 1, e1 (2012).

2. T. Barwicz, M. R. Watts, M. A. Popović, P. T. Rakich, L. Socci, F. X. Kärtner, E. P. Ippen, and H. I. Smith, Nat. Photonics 1, 57 (2007).

3. T. K. Liang and H. K. Tsang, Photon. Technol. Lett. 17, 393 (2005).

4. M. R. Watts, H. A. Haus, and E. P. Ippen, Opt. Lett. 30, 967 (2005).

5. H. Fukuda, K. Yamada, T. Tsuchizawa, T. Watanabe, H. Shinojima, and S. Itabashi, Opt. Express 14, 12401 (2006).

6. H. Zhang, Y. Huang, S. Das, C. Li, M. Yu, P. Lo, M. Hong, and J. Thong, Opt. Express 21, 3363 (2013).

7. J. Wang, D. Liang, Y. Tang, D. Dai, and J. Bowers, Opt. Lett. 38, 4 (2013).

8. S. Lin, J. Hu, and K. B. Crozier, Appl. Phys. Lett. 98, 151101 (2011).

9. D. Dai and J. Bowers, Opt. Express 19, 18614 (2011).

10. Q. Tan, X. Huang, W. Zhou, and K. Yang, Sci. Rep. 3, 2206 (2013).

11. C. Zou, F. Sun, C. Dong, X. Ren, J. Cui, X. Chen, Z. Han, and G. Guo, Opt. Lett. 36, 3630 (2011).

12. T. T. Le and M. C. Nguyen, J. Sci. Technol. 1, 8 (2012).

13. B. Rahman, N. Somasiri, C. Themistos, and K. Grattan, Appl. Phys. B 73, 613 (2001).

14. M. Yin, W. Yang, Y. Li, X. Wang, and H. Li, Opt. Commun. 335, 48 (2015).

15. X. Guan, H. Wu, Y. Shi, and D. Dai, Opt. Lett. 39, 259 (2014).

16. M. Z. Alam, X. Sun, M. Mojahedi, and J. S. Aitchison, Conference on Lasers and Electro-Optics, OSA Technical Digest (Optical Society of America, 2015) paper FW1D.2.

17. M. Z. Alam, X. Sun, M. Mojahedi, and J. S. Aitchison, "Augmented low index guiding: a new guiding mechanism for confining light in low index media," Laser Photon. Rev. (submitted).

18. D. Dai, Y. Tang, and J. E. Bowers, Opt. Express 20, 13425 (2012). 